\title{
ON THE APPROXIMATION TO INTEGRABLE FUNCTIONS BY INTEGRAL FUNCTIONS
}

\author{
BY \\ H. KOBER
}

1. Introduction. The problem of uniform approximation to a function $f(z)$ on some finite point set $D$ of the $z$-plane $(z=x+i y)$ (a) by polynomials and (b) by rational functions is classical. The theory has been summarized by J. L. Walsh $\left.{ }^{1}\right)$, who himself has proved a great number of important theorems some of which are fundamental.

Let $D$ be a point set containing the point $z=\infty$. In that case only problem (b) has a meaning; there is, however, another generalization of problem (a), the approximation to an arbitrary function, defined in $D$, by integral functions. Not much attention appears to have been paid to that problem up to now, except for the work of S. Bernstein $\left({ }^{2}\right)$.

Among the point sets $D$ extending to infinity the following are the simplest ones and appear to be of particular interest:

I. The real axis $-\infty<x<\infty, y=0$.

II. The positive part of the real axis $0<x<\infty, y=0$.

III. The half-plane $-\infty<x<\infty, y>0$.

IV. The strip $-\infty<x<\infty, a<y<b$.

V. The exterior of a circle.

In case $\mathrm{V}$; however, the uniform approximation by integral functions is not a reasonable problem, nor for any other domain of which $z=\infty$ is an interior point. In that case there is a circle $C$, with radius $R$ and center at the origin, such that the boundary of $D$ lies interior to $C$ and $C$ interior to $D$. Let $\left\{f_{n}(z)\right\}(n=1,2, \cdots)$ be a sequence of integral functions approximating to $f(z)$, then $\left|f(z)-f_{n}(z)\right| \leqq \epsilon_{n}$ for $|z| \geqq R$, where $\epsilon_{n} \rightarrow 0$ as $n \rightarrow \infty$. Hence $\left|f_{n}(z)-f_{1}(z)\right| \leqq \epsilon_{1}+\epsilon_{n}$, uniformly for $|z| \geqq R$, and, by the maximum-modulus theorem, for $|z|<R$. By the Liouville theorem, the function $f_{n}(z)-f_{1}(z)$ reduces to a constant, $c_{n}$, say, therefore $f(z)$ is an integral function, represented in the form $f(z)=f_{1}(z)+c+f_{n}(z)=c-c_{n}$, where $c=\lim c_{n}(n \rightarrow \infty)$.

Presented to the Society, April 24, 1943; received by the editors July 27, 1942.

(1) Interpolation and approximation by rational functions in the complex domain, Amer. Math. Soc. Colloquium Publications, vol. 20, New York, 1935.

(2) Leçons sur les propriêtés extrémales des fonctions analytiques d'une variable réelle, Paris, 1926. Bernstein gives some results concerning problem I; see Theorem $3(d), \S 6$, of the present paper. See, furthermore, the Bernstein Theorems 12 and 13 (pp. 105-107); they deal with sequences $\left\{f_{n}(z)\right\}$ of integral functions of exponential type, converging uniformly on the real axis to a function $f(z)$ of the same kind, or to a function $f(z)$ possessing some derivatives. A problem connected with the latter one has been treated by M. Krein, C. R. (Doklady) Acad. Sci. URSS. vol. 18 (1938) pp. 619-623. 
The cases I and II will be treated in the present paper, the cases III and IV in a further paper. We shall deal with approximation both in the sense of convergence in mean with index $p(0<p<\infty)$ and in the sense of uniform convergence $(p=\infty)$. The order of the approximating integral functions will be required to be finite and to be as small as possible. Whenever in an infinite $\left.{ }^{3}\right)$ interval a given function is approximated by integral functions of order $\rho$ then it can also be approximated by integral functions of any order $\rho^{\prime}>\rho(\S 5)$. The "best possible" value of $\rho$ is unity in problem $\mathrm{I}$, while it is one-half in problem II ( $\$ \S 6-8)$. Therefore the integral functions of finite types of the orders $\rho=1$ and $\rho=\frac{1}{2}$ play a dominant part in the results. Problem II presents the greatest difficulties. It will, in substance, be reduced to problem I, for $1<p<\infty$, however, by means of the approximation by rational functions $(\$ 7)$. The problem of "best approximation" will be treated in $\$ 9$, and a generalization of problem $I$ in $\$ 10$. A number of auxiliary results will be needed, some of which are possibly of interest in themselves. They will be proved first ( $\$ 2-4)$, in order to simplify the presentation of the main results.

I am greatly indebted to W. H. J. Fuchs, Aberdeen, in particular for suggesting to me the use of the Mittag-Leffler functions.

By $|f(t)|_{p}$ we denote

$$
\left(\int_{-\infty}^{\infty}|f(t)| p d t\right)^{1 / p} \text { or } \quad \underset{-\infty<t<\infty}{\text { ess. u.b. }}|f(t)|
$$

for $0<p<\infty$ or $p=\infty$, respectively; by $\|f(t)\|_{p}$ we denote

$$
\left(\int_{0}^{\infty}|f(t)|^{p} d t\right)^{1 / p} \text { or } \underset{\substack{\text { ess. u.b. } \\ 0<t<\infty}}{ }|f(t)| .
$$

By $G_{\beta}^{(\rho)}(0<\beta<\infty)$ we denote the set of integral functions $F(z)$ such that $|F(z)|<A_{\epsilon} e^{(\beta+\epsilon)|z| \rho}\left(A_{\epsilon}=A(\epsilon)\right)$ for any $\epsilon>0$, by $G^{(\rho)}$ the set of integral functions of order not greater than $\rho$. We write $G_{\beta}$ for $G_{\beta}^{(1)}$. Evidently $G_{\beta}^{(\rho)}>G_{\gamma}^{(\sigma)}$ when $0<\rho<\sigma$.

2. Generalization of a Hardy theorem and of a Plancherel-Pólya theorem.

Lemma 1. Let $0<p_{j}<\infty$, and let $f_{j}(z)$ be analyticfor $|z| \leqq R(j=1,2, \cdots, n)$. Then, for $0<r \leqq R$,

$$
W(r)=\int_{0}^{2 \pi} \sum_{j=1}^{n}\left|f_{j}\left(r e^{i \phi}\right)\right| p_{j} d \phi
$$

is a monotone increasing function of $r$, and $\log W(r)$ is a convex function of $\log r$.

For $n=1$ the result is due to G. H. Hardy $\left({ }^{4}\right)$. The general case can be de-

$\left({ }^{3}\right)$ In consequence of the Weierstrass theorem, in a finite interval a continuous function can be approximated uniformly by integral functions of any order.

(4) Proc. London Math. Soc. (2) vol. 14 (1915) pp. 269-277. 
duced by an argument due to G. Pólya and G. Szegö(5).

Lemma 2. Let $F(z) \in G_{\alpha}$ and $F(t)$ be bounded in $(-\infty, \infty)$. Then, for $-\infty<y<\infty,|F(x+i y)| \leqq e^{\alpha|y|}|F(t)|_{\infty}$.

Lemma 2'. Let $P(z)$ be a polynomial, let $F(z) \in G_{\alpha}$, let $0<p<\infty$ and $P(t)|F(t)|^{p} \in L_{1}(-\infty, \infty)$. Then

$$
\begin{gathered}
P(t)|F(t)|^{p} \rightarrow 0 \text { as } t \rightarrow \pm \infty \\
\int_{-\infty}^{\infty}|P(x+i y)||F(x+i y)|^{p} d x \leqq e^{\alpha p|y|} \int_{-\infty}^{\infty}|P(t)||F(t)|^{p} d t .
\end{gathered}
$$

For $0<y<\infty$, Lemma 2 is proved by applying a Phragmén-Lindelöf theo$\operatorname{rem}\left({ }^{6}\right)$ to the function $e^{i(\alpha+\epsilon) z} F(z)(\epsilon>0)$ first in both quadrants of the upper half-plane, and then in the upper half-plane, and finally by letting $\epsilon \rightarrow 0$. For $P(z) \equiv 1$, Lemma $2^{\prime}$ has been proved by M. Plancherel and G. Pólya $\left({ }^{7}\right)$; the general case can be deduced in a similar way, starting from the inequality

$$
\left|P\left(z_{0}\right)\right|\left|\Phi\left(z_{0}\right)\right|^{p} \leqq \frac{1}{2 \pi} \int_{0}^{2 \pi}\left|P\left(z_{0}+r e^{i \phi}\right)\right|\left|\Phi\left(z_{0}+r e^{i \phi}\right)\right|^{p} d \phi,
$$

where $\Phi(z)$ is regular for $\left|z-z_{0}\right| \leqq r$. This inequality is a simple application of Lemma 1.

3. On the spaces $G_{\alpha, p}$ and $G_{\alpha, p}^{(1 / 2)}$. Let $p$ and $\alpha$ be fixed $(0<p \leqq \infty, 0<\alpha<\infty)$. By $G_{\alpha, p}$ or $G_{\alpha, p}^{(1 / 2)}$ we denote the set of functions $f(z)$, belonging to $G_{\alpha}$ or $G_{\alpha}^{(1 / 2)}$ and such that $f(t) \in L_{p}(-\infty, \infty)$ or $L_{p}(0, \infty)$, respectively.

Lemma $3\left(^{8}\right)$. Let $f_{n}(z) \in G_{\alpha, p}(n=1,2, \cdots)$ and $\left|f_{m}(t)-f_{n}(t)\right|_{p} \rightarrow 0$ as $m>n \rightarrow \infty$. Then there is an element $f(z)$ of $G_{\alpha, p}$ such that $\left|f(t)-f_{n}(t)\right|_{p} \rightarrow 0$ as $n \rightarrow \infty$.

Lemma $3^{\prime}$. The preceding result holds when $G_{\alpha, p}$ is replaced by $G_{\alpha, p}^{(1 / 2)}$, and $\left|f_{m}(t)-f_{n}(t)\right|_{p} b y\left\|f_{m}(t)-f_{n}(t)\right\|_{p},\left|f(t)-f_{n}(t)\right|_{p} b y\left\|f(t)-f_{n}(t)\right\|_{p}$.

The lemmas do not hold whenever the order $\rho$ of the integral functions is greater than 1 or $\frac{1}{2}$, respectively $\left({ }^{9}\right)$.

(5) Aufgaben und Lehrsätze aus der Analysis, Berlin, 1925, vol. I, pp. 143, 144, nos. 302, 310. We start from the lemma: Let $f_{i, k}(z)(j=1,2, \cdots, m ; k=1,2, \cdots, n)$ be regular and $\left|f_{j, k}(z)\right|$ be one-valued in the closed domain $D$, let $0<p_{i}<\infty$. Then the function $\sum_{k=1}^{n}\left|f_{1, k}(z)\right| p_{1}\left|f_{2, k}(z)\right| p_{2}$ $\cdots\left|f_{m, k}(z)\right| p_{m}$ attains its maximum value on the boundary of $D$.

(6) E.g. E. C. Titchmarsh, The theory of functions, Oxford, 1932, p. 177.

(7) Comment. Math. Helv. vol. 10 (1937-1938), §\$27-30.

(8) For $p=\infty$, the result is equivalent to the Bernstein Theorem 13.

(9) Let $\rho>1$ or $\rho>\frac{1}{2}$, respectively, be fixed, let $f(t)$ be continuous for $-1 \leqq t \leqq 1$ and $f(t)=0$ for $|t| \geqq 1$. Then $f(t)$ is not equivalent to an integral function in $(-\infty, \infty)$ nor in $(0, \infty)$. On the other hand, by Theorem $3(\mathrm{~b}), \S 6$, and by the argument of $\$ 5$, there are integral functions $g_{n}(z)$ of the type unity of the order $\rho$, approximating to $f(t)$ in $(-\infty, \infty)$ or $(0, \infty)$, respectively, as $n \rightarrow \infty$; therefore $\left|g_{m}(t)-g_{n}(t)\right|_{p \rightarrow 0}$ or $\left\|g_{m}(t)-g_{n}(t)\right\|_{p} \rightarrow 0$. For $p=\infty$ and $\frac{1}{2}<\rho \leqq 1$, how- 
Let $f_{n}(z) \in G_{\alpha, p}$. In consequence of the hypothesis, there is a constant $A$, independent of $n$, such that $\left|f_{n}(t)\right|_{p}<A$, and a function $\Phi(t) \in L_{p}(-\infty, \infty)$ such that $\left|\Phi(t)-f_{n}(t)\right|_{p} \rightarrow 0$ as $n \rightarrow \infty$. Take first $p=\infty$. By Lemma 2, we have $\left|f_{n}(z)\right|<A e^{\alpha|y|}$,

$$
\left|f_{m}(z)-f_{n}(z)\right| \leqq e^{\alpha|y|}\left|f_{m}(t)-f_{n}(t)\right|_{\infty} .
$$

Consequently the $f_{n}(z)$ converge to an analytic function $f(z)$, uniformly in any finite domain of the z-plane. Therefore, for any value of $z$, we have $|f(z)| \leqq A e^{\alpha|y|}$, and so $f(z) \in G_{\alpha}$. Since, uniformly in any finite interval $(-M, M), f(t)-f_{n}(t) \rightarrow 0$ as $n \rightarrow \infty$, we have $f(t) \equiv \Phi(t)$ in $(-M, M)$ and, therefore, in $(-\infty, \infty)$.

Let now $0<p<\infty$, and put $f_{m, n}=f_{m}-f_{n}$. By an argument due to Plancherel and Pólya $\left({ }^{10}\right)$, we have

$$
\begin{aligned}
\left|f_{m, n}(x)\right|^{p} & \leqq \frac{1}{\pi} \int_{-1}^{1} d \tau \int_{-1}^{1}\left|f_{m, n}(x+t+i \tau)\right|^{p} d t \\
& \leqq \frac{1}{\pi} \int_{-1}^{1} d \tau \int_{-\infty}^{\infty}\left|f_{m, n}(s+i \tau)\right|^{p} d s .
\end{aligned}
$$

Using Lemma $2^{\prime}(\mathrm{b})$, with $P(t) \equiv 1$, we have

$$
\left|f_{m, n}(x)\right| \leqq(2 / \pi)^{1 / p} e^{\alpha}\left|f_{m, n}(t)\right|_{p} \quad(-\infty<x<\infty) .
$$

By the argument used in the first part of the proof we deduce the existence of a function $f(z)$ belonging to $G_{\alpha}$ and such that, in any finite interval $(-M, M)$,

$$
\int_{-M}^{M}\left|f(t)-f_{n}(t)\right|^{p} d t \rightarrow 0 \text { as } n \rightarrow \infty \text {. }
$$

Hence $f(t) \equiv \Phi(t)$ in $(-M, M)$ and, therefore, in $(-\infty, \infty)$.

The case $f_{n}(z) \in G_{\alpha}^{(1 / 2)}$ is more difficult. Taking $F_{n}(z)=f_{n}\left(z^{2}\right), F_{n}(z)$ is an even function and belongs to $G_{\alpha}$. By this transformation, the result for $p=\infty$ is readily deduced from the corresponding result on $G_{\alpha}$. Let now $0<p<\infty$, and $F_{n}(z)=f_{n}\left(z^{2}\right)$. Then we have

$$
\begin{gathered}
\left\|f_{n}(t)\right\|_{p}=\left.\left.\left|F_{n}(t)\right| t\right|^{1 / p}\right|_{p}<A \quad(n=1,2, \cdots) . \\
\int_{0}^{\infty}\left|f_{m}(t)-f_{n}(t)\right|^{p} d t=\int_{-\infty}^{\infty}\left|F_{m}(t)-F_{n}(t)\right| p|t| d t .
\end{gathered}
$$

We have to prove the existence of an even function $F(z) \in G_{\alpha}$ such that $\left.\left.\left|\left\{F(t)-F_{n}(t)\right\}\right| t\right|^{1 / p}\right|_{p} \rightarrow 0$ as $n \rightarrow \infty$. In consequence of the hypothesis, there

ever, we can prove: Let $f_{n}(z) \in G_{\alpha}^{()}$, let $\gamma=2 \pi-\pi \rho^{-1}$, let $C$ consist of two straight lines starting at the origin and making the angle $\gamma$ between them, and let, uniformly for $z$ on $C, f_{m}(z)-f_{n}(z) \rightarrow 0$ as $m>n \rightarrow \infty$. Then there is an element $f(z)$ of $G_{\alpha}^{(\rho)}$ such that, uniformly on $C, f_{n}(z) \rightarrow f(z)$ as $n \rightarrow \infty$.

$\left.{ }^{10}\right)$ Loc. cit., $\$ 30(73)$. The proof is based on the Hardy theorem. 
is an even function $\Phi(t)$ such that $\Phi(t)|t|^{1 / p} \in L_{p}(-\infty, \infty)$ and that $\left.|\phi(t)| t\right|^{1 / p}-\left.F_{n}(t)|t|^{1 / p}\right|_{p} \rightarrow 0$ as $n \rightarrow \infty$. Using the Plancherel-Pólya argument and Lemma $2^{\prime}(\mathrm{b})$, we have

$$
|z|\left|F_{n}(z)\right|^{p} \leqq \frac{1}{\pi} \int_{-1}^{1} d \tau \int_{-1}^{1}|z+t+i \tau|\left|F_{n}(z+t+i \tau)\right|^{p} d t
$$

Similarly

$$
\leqq \frac{1}{\pi} \int_{-1}^{1} d \tau e^{\alpha p|y+\tau|} \int_{-\infty}^{\infty}\left|F_{n}(t)\right| p|t| d t \leqq \frac{2}{\pi} e^{\alpha p(1+|y|)}\left\|f_{n}(t)\right\|_{p}^{p} .
$$

$$
|z|\left|F_{m}(z)-F_{n}(z)\right|^{p} \leqq(2 / \pi) e^{\alpha p(1+|y|)}\left\|f_{m}(t)-f_{n}(t)\right\|_{p}^{p} .
$$

Hence, if $k$ is an integer greater than $p^{-1}$ and $M$ any positive number, we have $z^{k} F_{m}(z)-z^{k} F_{n}(z) \rightarrow 0$ as $m>n \rightarrow \infty$, uniformly for $-M \leqq x \leqq M,-M \leqq y \leqq M$. Consequently $z^{k} F_{n}(z)$ tends to an analytic function $\Psi(z)$ as $n \rightarrow \infty$, uniformly in any finite domain of the $z$-plane. By (3.5) and (3.3), there exists a constant $A^{\prime}$, independent of $n$, such that $|z|\left|F_{n}(z)\right|^{p} \leqq A^{\prime}$ for $|z| \leqq 1$. By the maximum-modulus theorem, we have $\left|F_{n}(z)\right|^{p} \leqq A^{\prime}$ for $|z| \leqq 1$. Therefore, for $|z| \leqq 1$, we have $|\Psi(z)| \leqq A^{\prime}|z|^{k}$ which shows that $F(z)=z^{-k} \Psi(z)$ is an integral function. Since $z^{k} F_{n}(z) \rightarrow \Psi(z)$, from (3.5) and (3.3) we deduce that $\Psi(z)$ and, therefore, $F(z)$ belong to $G_{\alpha}$. We are left to show that $F(t) \equiv \Phi(t)$ in $(-\infty, \infty)$. Let $M$ be any positive number, and $E$ be the set consisting of the two intervals $\left(-M,-M^{-1}\right)$ and $\left(M^{-1}, M\right)$. By the uniform convergence of the sequence $\left\{z^{k} F_{n}(z)\right\}$, we have

$$
\int_{E}\left|F(t)-F_{n}(t)\right|^{p}|t| d t=\int_{E}\left|\Psi(t)-t^{k} F_{n}(t)\right|^{p}|t|^{1-p k} d t \rightarrow 0
$$

as $n \rightarrow \infty$. Hence $F(t) \equiv \Phi(t)$ in $E$ and, therefore, in $(-\infty, \infty)$, and $F(t)$ is an even function; so is, therefore, $F(z)$.

We remark that, in the terminology of S. Banach, $G_{\alpha, p}$ and $G_{\alpha, p}^{(1 / 2)}$ are $(B)$ spaces for $1 \leqq p \leqq \infty\left({ }^{11}\right)$. For $0<p<1$, they are $(F)$ spaces if the distance of an element $f(z)$ from the null-element is defined by $|f(t)|_{p}^{p}$ or $\|f(t)\|_{p}^{p}$, respectively.

\section{Further basic lemmas.}

Lemma 4. Let $\kappa(z) \in G_{1}$. Let $1 \leqq p \leqq \infty, 1 \leqq q \leqq \infty, p^{-1}+q^{-1} \geqq 1$ and $f(t) \in L_{p}(-\infty, \infty), \kappa(t) \in L_{q}(-\infty, \infty)$. Let

$$
g_{\alpha}(z)=\alpha \int_{-\infty}^{\infty} \kappa\{\alpha(t-z)\} f(t) d t \quad(z=x+i y) .
$$

Then $g_{\alpha}(z)$ belongs to $G_{\alpha}$.

Proof. Let $p^{\prime}=p /(p-1)$. Then we have $p^{\prime} \geqq q$ and, by Lemma $2^{\prime}(\mathrm{a})$, $\kappa(t) \in L_{p^{\prime}}(-\infty, \infty)$. By Lemma $2^{\prime}(\mathrm{b})$, we have

(11) With "norm" $|f(t)|_{p}$ or $\|f(t)\|_{p}$. S. Banach, Thêorie des opérations linéaires, Warsaw, 1932, Chapters III and IV. 


$$
|\kappa\{\alpha t-\alpha(x+i y)\}|_{p^{\prime}}=|\kappa(\alpha t-\alpha i y)|_{p^{\prime}} \leqq \alpha^{-1 / p^{\prime}} e^{\alpha|y|}|\kappa(t)|_{p^{\prime}}
$$

Therefore, by Hölder's theorem, from (4.1) we deduce that

$$
\left|g_{\alpha}(z)\right| \leqq \alpha^{1 / p} e^{\alpha|y|}|\kappa(t)|_{p^{\prime}}|f(t)|_{p}<A e^{\alpha|y|} .
$$

Let $C$ be the circle $\zeta=2 R e^{i \phi}(0 \leqq \phi \leqq 2 \pi)$, and let $|z| \leqq R$. Then

$$
\begin{aligned}
\frac{1}{2 \pi i} \int_{C} \frac{g_{\alpha}(\zeta)}{\zeta-z} d \zeta & =\frac{\alpha}{2 \pi i} \int_{-\infty}^{\infty} f(t) d t \int_{C} \frac{\kappa\{\alpha(t-\zeta)\}}{\zeta-z} d \zeta \\
& =\alpha \int_{-\infty}^{\infty} \kappa\{\alpha(t-z)\} f(t) d t=g_{\alpha}(z),
\end{aligned}
$$

the in terchange of integrations being justified by absolute convergence. Hence $g_{\alpha}(z)$ is an analytic function for $|z| \leqq R$. Since $R$ is arbitrary, $g_{\alpha}(z)$ is an integral function, which completes the proof.

Lemмa 5. Let $k$ be a positive integer, let $\kappa(z) \in G_{1}$ and $t^{k} \kappa(t) \in L_{1}(-\infty, \infty)$. Let $f(t)$ be measurable and bounded in any finite interval and $f(t)=O\left(|t|^{k}\right)$ $(t \rightarrow \pm \infty)$. Then the function $g_{\alpha}(z)$, defined by (4.1), belongs to $G_{\alpha}$.

Obviously $\kappa(t) \in L_{1}(-\infty, \infty)$. We have $|f(t)| \leqq A\left(1+|t|^{k}\right) \leqq A^{\prime}\left(1+|z|^{k}\right.$ $\left.+|t-z|^{k}\right)$ for $-\infty<t<\infty$. Therefore, taking $\kappa_{0}(z)=z^{k} \kappa(z)$, we deduce that

$$
\begin{aligned}
\left|g_{\alpha}(z)\right| \leqq & \alpha^{1-k} A^{\prime} \int_{-\infty}^{\infty}\left|\kappa_{0}\{\alpha(t-z)\}\right| d t \\
& +A^{\prime}\left(|z|^{k}+1\right) \int_{-\infty}^{\infty}|\kappa\{\alpha(t-z)\}| d t, \\
\left|g_{\alpha}(z)\right| \leqq & A^{\prime \prime}\left(1+|z|^{k}\right) e^{\alpha|y|},
\end{aligned}
$$

using (4.2), with $p^{\prime}=1$. It is easy to show that (4.4) holds which completes the proof.

Lemma 6. Let $1 \leqq q<\infty$, let $k-1$ be a positive integer and $p=q k^{-1}$, let $\phi(x)$, $\psi(x) \in L_{q}(-\infty, \infty)$ and $M=\max \left\{|\phi(x)|_{q},|\psi(x)|_{q}\right\}$. Then

$$
\left|\{\phi(x)\}^{k}-\{\psi(x)\}^{k}\right|_{p} \leqq k^{\lambda} M^{k-1}|\phi(x)-\psi(x)|_{q}(\lambda=\max (1,(k-1) / q)) .
$$

Proof. Applying Hölder's inequality, we have

$$
\begin{aligned}
\int_{-\infty}^{\infty}\left|\phi^{k}-\psi^{k}\right|{ }^{p} d x & =\int_{-\infty}^{\infty}\left(|\phi-\psi|\left|\sum_{j=0}^{k-1} \phi^{j} \psi^{k-1-j}\right|\right)^{p} d x \\
& \leqq|\phi-\psi|_{q}^{p}\left|\sum_{j=0}^{k-1} \phi^{j} \psi^{k-1-j}\right|_{q /(k-1)}^{p}, \\
\left|\phi^{j} \psi^{k-1-j}\right|_{q /(k-1)} & \leqq\left(|\phi|_{q}^{j /(k-1)}|\psi|_{q}^{1-j /(k-1)}\right)^{k-1} \leqq M^{k-1},
\end{aligned}
$$

which gives the required result. Obviously it holds in $(0, \infty)$. 


\section{Preliminary approximation theorems.}

Theorem 1. Let $0<p \leqq \infty$, let $f(t)$ belong to $L_{p}(-\infty, \infty)$ or $L_{p}(0, \infty)$, let $\rho<1$ or $\rho<\frac{1}{2}$, respectively. Let $f(t)$ be approximated by elements $g_{n}(z)$ of $G^{(\rho)}$ $(n=1,2, \cdots)$, that is,

$$
\left|f(t)-g_{n}(t)\right|_{p} \rightarrow 0 \text { or }\left\|f(t)-g_{n}(t)\right\|_{p} \rightarrow 0 \text { as } n \rightarrow \infty
$$

for $\rho<1$ or $\rho<\frac{1}{2}$, respectively. Then $f(t)$ reduces to a constant which is zero for $0<p<\infty$.

I. The interval $(-\infty, \infty)$. Here the theorem is a corollary of a result due to V. G. Iyer $\left.{ }^{12}\right)$. It can also be deduced from the Lemmas $2^{\prime}(a)$ and 2 by means of the Liouville theorem.

II. The interval $(0, \infty)$. Since $\rho<\frac{1}{2}$, we have $g_{n}(z) \in G_{0}^{(1 / 2)}$. Hence the even function $F(z)=g_{n}\left(z^{2}\right)$ belongs to $G_{0}$. Since $g_{n}(t) \in L_{p}(0, \infty)$, we have $|t|^{1 / p} F_{n}(t) \in L_{p}(-\infty, \infty)$ and, therefore, $F_{n}(t) \in L_{p}(-\infty, \infty)$. We can again employ the Lemmas $2^{\prime}(a)$ and 2, which proves the theorem.

THEOREM 2. Let $0<p \leqq \infty$ and let $f(t)$ belong to $L_{p}(-\infty, \infty)$ or $L_{p}(0, \infty)$, respectively, let $f(t)$ be approximated by elements $g_{n}(z)$ of $G^{(\rho)}(n=1,2, \cdots)$, and let $\sigma$ be any number greater than $\rho$. Then $f(t)$ can be approximated by integral functions of order $\sigma$.

I. The interval $(-\infty, \infty)$. By Theorem 1 , we may suppose that $\rho \geqq 1$, $\sigma>1$. We need only find a single integral function $W(z)$ of order $\sigma$ such that $W(t) \in L_{p}(-\infty, \infty)(0<p \leqq \infty)$. For (5.1) implies that $\left|f(t)-h_{n}(t)\right|_{p} \rightarrow 0$ as $n \rightarrow \infty$, where $h_{n}(z)=g_{n}(z)+n^{-1} W(z)$, and the integral function $h_{n}(z)$ is of $\operatorname{order} \sigma$.

Let $0<\lambda<1$. The Mittag-Leffler function

$$
E_{\lambda}(z)=\sum_{n=0}^{\infty} \frac{z^{n}}{\Gamma(1+\lambda n)}
$$

is well known to be an integral function of the type unity of the order $\lambda^{-1}$. Let $0<\epsilon<1-\lambda$. Then, uniformly in the angle $(\pi / 2)(\lambda+\epsilon) \leqq \arg z \leqq 2 \pi$ $-(\pi / 2)(\lambda+\epsilon)$, we have

$$
E_{\lambda}(z)=O\left(|z|^{-1}\right) \quad(|z| \rightarrow \infty) .
$$

When $k$ is any integer greater than $p^{-1}$ then the function $W(z)=\left(E_{1 / \sigma}(i z)\right)^{k}$ has the required property.

II. The interval $(0, \infty)$. It will suffice to take $\sigma>1 / 2$. The integral function $W(z)=\left(E_{1 / \sigma}(-\dot{z})\right)^{k}$ is an integral function of order $\sigma$, and $W(t) \in L_{p}(0, \infty)$, which proves the theorem.

(12) Quart. J. Math. Oxford Ser. vol. 7 (1936) pp. 294-299, Theorems 2 and 4. 
6. Approximation functions of lowest order. Let $0<p \leqq \infty$ and $f(t) \in L_{p}(-\infty, \infty)$, and let $f(t)$ not reduce to a constant. By Theorems 1 and 2 there exists a lower bound of the $\rho$ 's, provided that $f(t)$ can be approximated at all by integral functions of finite order. This minimum is in fact attained.

Theorem 3(a). Let $0<p<\infty$ and $f(t)$ belong to $L_{p}(-\infty, \infty)$ or $L_{p}(0, \infty)$, respectively. Then there are functions $g_{\alpha}(z) \in G_{\alpha}$ or $\in G_{\alpha}^{(1 / 2)}(0<\alpha \rightarrow \infty)$ such that

$$
\left|f(t)-g_{\alpha}(t)\right|_{p} \rightarrow 0 \text { or }\left\|f(t)-g_{\alpha}(t)\right\|_{p} \rightarrow 0 \text { as } \alpha \rightarrow \infty .
$$

Theorem 3(b). Let $f(t)$ belong to $L_{\infty}(-\infty, \infty)$ or $L_{\infty}(0, \infty)$, respectively. $A$ necessary and sufficient condition for the existence of functions $g_{\alpha}(z) \in G_{\alpha}$ or $\in G_{\alpha}^{(1 / 2)}(0<\alpha \rightarrow \infty)$ such that

$$
\left|f(t)-g_{\alpha}(t)\right|_{\infty} \rightarrow 0 \text { or }\left\|f(t)-g_{\alpha}(t)\right\|_{\infty} \rightarrow 0 \text { as } \alpha \rightarrow \infty
$$

is that $f(t)$ or $f\left(t^{2}\right)$, respectively, is uniformly continuous in $(-\infty, \infty)$.

The following theorems concern classes of functions $f(t)$ which need not belong to any $L_{p}(-\infty, \infty)(0<p \leqq \infty)$.

THEOREM 3(c). Let, for some $r(0<r \leqq 1)$, and uniformly for $-\infty<t_{j}<\infty$ $(j=1,2), f(t)$ satisfy the Lipschitz condition

$$
\left|f\left(t_{1}\right)-f\left(t_{2}\right)\right| \leqq A\left|t_{1}-t_{2}\right|^{r}
$$

Then $f(t)$ can be approximated by functions $g_{\alpha}(z) \in G_{\alpha}(0<\alpha \rightarrow \infty)$, uniformly in $(-\infty, \infty)$.

TheOREM 3(d). (Bernstein's theorem) $\left({ }^{13}\right)$. The preceding result holds if $f(t)$ is continuous and $f(t)-f_{1}(t) \rightarrow 0$ as $t \rightarrow \infty$ and $f(t)-f_{2}(t) \rightarrow 0$ as $t \rightarrow-\infty$, where $f_{1}(t)$ and $f_{2}(t)$ are linear functions.

7. Proofs of Theorems 3.

I. The interval $(-\infty, \infty)$. We start from the following result $\left({ }^{14}\right)$.

Lemma 7. Let $f(t) \in L_{p}(-\infty, \infty)(1 \leqq p<\infty)$, or let $f(t)$ belong to $L_{\infty}(-\infty, \infty)$ and be uniformly continuous in $(-\infty, \infty)$; let $\kappa(t) \in L_{1}(-\infty, \infty)$ and $\int_{-\infty}^{\infty} \kappa(t) d t$ $=1$, and let $g_{\alpha}(x)$ be defined by (4.1), replacing $z$ by $x$. Then, as $\alpha \rightarrow \infty$, $\left|f(t)-g_{\alpha}(t)\right|_{p \rightarrow 0}(1 \leqq p \leqq \infty)$.

Let $\kappa(z)$ satisfy the conditions both of Lemma 4 and of Lemma 7. Take, for instance, the Féjer kernel $\kappa(z)=\left(\pi z^{2}\right)^{-1}(1-\cos z)$. Then Theorem 3(a), for $1 \leqq p<\infty$, and the sufficiency of the conditions in Theorem $3(b)$ is an immediate consequence of the lemma. The necessity of the condition in $3(b)$ is readily deduced from the Bernstein $\left({ }^{15}\right)$ theorem:

(13) Loc. cit., 106, footnote. It can be deduced from Theorems 3(b) and (c).

(14) E. Hille, Trans. Amer. Math. Soc. vol. 39 (1936) pp. 131-153 §1.

(15) Loc. cit., 102, Corollary 7. 
Lemma 8. Let $g(z) \in G_{\alpha}$ and $|g(t)| \leqq A$ in $(-\infty, \infty)$. Then $\left|g^{\prime}(t)\right| \leqq \alpha A$ in $(-\infty, \infty)$, therefore $g(t)$ is uniformly continuous in $(-\infty, \infty)$.

Let now $0<p<1$ and $f(t) \in L_{p}(-\infty, \infty)$. Let $k$ be an integer not smaller than $p^{-1}$ and $k p=q$. Defining $\{f(t)\}^{1 / k}$ in a suitable way, we have $\{f(t)\}^{1 / k}$ $\in L_{q}(-\infty, \infty)$. Let $\kappa(z)$ be defined as, above, and let

$$
g_{\alpha}(z)=\left(\frac{\alpha}{k} \int_{-\infty}^{\infty}{ }^{\kappa}\left(\frac{\alpha t}{k}-\frac{\alpha z}{k}\right)\{f(t)\}^{1 / k} d t\right)^{k}=\left\{\gamma_{\beta}(z)\right\}^{k},
$$

where $\beta=\alpha k^{-1}$. Then $\left|f(t)-g_{\alpha}(t)\right|_{p} \rightarrow 0$ as $\alpha \rightarrow \infty$. Obviously $\gamma_{\beta}(z) \in G_{\beta}$, and $\epsilon_{\beta}=\left|\{f(t)\}^{1 / k}-\gamma_{\beta}(t)\right|_{q} \rightarrow 0$ as $\beta \rightarrow \infty$. By Lemma 6, we have

$$
\left|f(t)-\left\{\gamma_{\beta}(t)\right\}^{k}\right|_{p} \leqq A\left|\{f(t)\}^{1 / k}-\gamma_{\beta}(t)\right|_{q} \rightarrow 0 \text { as } \beta \rightarrow \infty,
$$

since

$$
\max \left(\left|f^{1 / k}\right|_{q},\left|\gamma_{\beta}(t)\right|_{q}\right) \leqq\left|f^{1 / k}\right|_{q}+\epsilon_{\beta}=|f(t)|_{p}^{1 / k}+\epsilon_{\beta}<A^{\prime} .
$$

Hence $\left|f(t)-g_{\alpha}(t)\right|_{p} \rightarrow 0$ as $\alpha \rightarrow \infty$, and obviously $g_{\alpha}(z) \in G_{\alpha}$.

To prove Theorem $3(\mathrm{c})$, we take, for instance,

$$
\kappa(z)=a z^{-4} \sin ^{4} \frac{z}{4}, \quad a^{-1}=\int_{-\infty}^{\infty} \frac{d t}{t^{4}} \sin ^{4} \frac{t}{4}=\frac{\pi}{96} .
$$

Since $\kappa(z)$ and, by (6.1), $f(t)$, with $k=1$, satisfy the hypotheses of Lemma 5, the function $g_{\alpha}(z)$, defined by (4.1), belongs to $G_{\alpha}$. We have

$$
f(x)-g_{\alpha}(x)=\alpha \int_{-\infty}^{\infty} \kappa(\alpha t)\{f(x)-f(t+x)\} d t .
$$

Hence, by (6.1),

$$
\left|f(x)-g_{\alpha}(x)\right| \leqq A \alpha \int_{-\infty}^{\infty}|\kappa(\alpha t)||t|{ }^{r} d t=A \alpha^{-r} \int_{-\infty}^{\infty}\left|\kappa(t) t^{r}\right| d t,
$$

which tends to zero as $\alpha \rightarrow \infty$, uniformly for $-\infty<x<\infty$. This completes the proof of the assertions concerning the interval $(-\infty, \infty)$.

As an application of Theorem 3(c) we obtain the following result:

Let $0<r<1$, and let $z^{r}$ be uniquely defined by $-\pi / 2 \leqq \arg z \leqq 3 \pi / 2$. Then $t^{r}$ can be approximated by functions $g_{\alpha}(z) \in G_{\alpha}(0<\alpha \rightarrow \infty)$, uniformly in $(-\infty, \infty)$.

The result follows immediately from the inequality $\left|t_{1}^{r}-t_{2}^{r}\right|^{0} \leqq 2^{1-r}\left|t_{1}-t_{2}\right|^{r}$. It holds for $0<r<\infty$, and the corresponding result holds for the interval $(0, \infty)$, with $g_{\alpha}(z) \in G_{\alpha}^{(1 / 2)}$; this will be shown in the paper indicated above. We remark that, for $r \neq 1,2,3, \cdots, t^{r}$ cannot be approximated by rational functions in $(-\infty, \infty)$ or $(0, \infty)$.

II. The interval $(0, \infty)$. This case is, for $p=\infty$, readily deduced from the corresponding result for the interval $(-\infty, \infty)$. We have to take into 
consideration that $F(t)=f\left(t^{2}\right)$ is an even function and, therefore,

$$
\gamma_{\alpha}(z)=\frac{1}{\pi \alpha} \int_{-\infty}^{\infty} \frac{1-\cos \alpha(t-z)}{(t-z)^{2}} F(t) d t
$$

is an even function as well. Let now $p=1$ and again $F(t)=f\left(t^{2}\right)$. Then $t F(t) \in L_{1}(-\infty, \infty)$. Replacing $F(t)$ by $t F(t)$ in (7.1), we see that $\gamma_{\alpha}(z)$ is an odd function, and that $\left|t F(t)-\gamma_{\alpha}(t)\right|_{1 \rightarrow 0}$ as $\alpha \rightarrow \infty$. Taking $g_{\alpha}(z)=z^{-1 / 2} \gamma_{\alpha}\left(z^{1 / 2}\right)$, we have $g_{\alpha}(z) \in G_{\alpha}^{(1 / 2)}$ and $\left\|f(t)-g_{\alpha}(t)\right\|_{1} \rightarrow 0$ as $\alpha \rightarrow \infty$.

For the further investigation, we need

Lemma 9(16). The sequence $\left\{\pi^{-1 / 2}(i-t)^{n}(i+t)^{-n-1}\right\}(n=0, \pm 1, \pm 2, \cdots)$ is a complete orthonormal system with respect to $L_{p}(-\infty, \infty)$ for $1<p<\infty$.

Consequently $\left({ }^{16}\right)$, for $1<p<\infty$, any element $f(t) \in L_{p}(0, \infty)$, can certainly be approximated, in the mean of order $p$, by finite linear forms of the $(i-t)^{n}(i+t)^{-n-1}$. Therefore we need only show, that, given $\epsilon>0$ and any integer $n$, there is an element $g_{\alpha}(z) \in G_{\alpha}^{(1 / 2)}$ such that $\left\|(i-t)^{n}(i+t)^{-n-1}-g_{\alpha}(t)\right\|_{p}$ $<\epsilon$, or that there exists an even function $\gamma_{\alpha}(z) \in G_{\alpha}$ such that

$$
\int_{-\infty}^{\infty}\left|\frac{\left(i-t^{2}\right)^{n}}{\left(i+t^{2}\right)^{n+1}}-\gamma_{\alpha}(t)\right|^{p}|t| d t<\epsilon^{p} .
$$

Let $\kappa(z)$ be defined by (7.2), and let

$$
g_{\alpha}(z)=\alpha \int_{-\infty}^{\infty} \kappa\{\alpha(t-z)\} \frac{\left(i-t^{2}\right)^{n}}{\left(i+t^{2}\right)^{n+1}} d t .
$$

Then, for $\alpha \geqq A=A(\epsilon)$, we have

$$
\left|\frac{\left(i-t^{2}\right)^{n}}{\left(i+t^{2}\right)^{n+1}}-g_{\alpha}(t)\right|_{p}<\epsilon .
$$

The function $g_{\alpha}(z)$ is even, and we have

$$
\begin{aligned}
x g_{\alpha}(x) & =-\int_{-\infty}^{\infty} \kappa_{0}\{\alpha(t-x)\} \frac{\left(i-t^{2}\right)^{n} d t}{\left(i+t^{2}\right)^{n+1}}+\alpha \int_{-\infty}^{\infty} \kappa\{\alpha(t-x)\} \frac{t\left(i-t^{2}\right)^{n}}{\left(i+t^{2}\right)^{n+1}} d t \\
& =\phi_{\alpha}(x)+\psi_{\alpha}(x),
\end{aligned}
$$

where $\kappa_{0}(z)=z \kappa(z)$. Obviously $\left|t\left(i-t^{2}\right)^{n}\left(i+t^{2}\right)^{-n-1}-\psi_{\alpha}(t)\right|_{p \rightarrow 0}$ as $\alpha \rightarrow \infty$, while, by a well known convexity theorem $\left({ }^{17}\right)$,

$$
\left|\phi_{\alpha}(x)\right|_{p} \leqq \alpha^{-1}\left|\kappa_{0}(t)\right|_{1}\left|\left(i+x^{2}\right)^{-1}\right|_{p}
$$

(16) H. Kober, a forthcoming paper in Quart. J. Math. Oxford Ser. For the case $p=2$, see also E. Hille, Compositio Math. vol. 6 (1938) p. 99.

(17) E.g., Inequalities, G. H. Hardy, J. E. Littlewood and G. Polya, Cambridge, 1934, Theorem 204. 
Hence $\left|\phi_{\alpha}(x)\right|_{p} \rightarrow 0$ as $\alpha \rightarrow \infty$; consequently, for some $\alpha>A$, we have

$$
\left|\left(i-t^{2}\right)^{n} t\left(i+t^{2}\right)^{-n-1}-\operatorname{tg}_{\alpha}(t)\right|_{p}<\epsilon .
$$

By Hölder's inequality, from (7.5) and (7.6) we deduce (7.4). For, taking $\chi(t)=\left(i-t^{2}\right)^{n}\left(i+t^{2}\right)^{-n-1}-g_{\alpha}(t)$, we have

$$
\int_{-\infty}^{\infty}|\chi|^{p}|t| d t=\int_{-\infty}^{\infty}|t \chi||\chi|^{p-1} d t \leqq|t \chi|_{p}|\chi|_{p}^{p-1}<\epsilon^{p}
$$

Thus we have proved the theorem for $1 \leqq p<\infty$. The case $0<p<1$ is treated as the corresponding case in $(-\infty, \infty)$.

8. Uniqueness. By the argument used in Theorem 2, it is evident that the sets of approximating functions, occurring in Theorems 3(a)-(c), are not at all unique. We can construct approximating functions of order $\rho=1$ or $\rho=\frac{1}{2}$, respectively, with types tending to infinity, even when $f(t)$ reduces to a null-function. Certainly for $f(t) \in L_{p}(-\infty, \infty)$ or $\in L_{p}(0, \infty)$, however, the types tend necessarily to infinity provided that $f(t)$ is not equivalent to a constant or to an integral function of finite type of the order $\rho=1$ or $\rho=\frac{1}{2}$, respectively. We can prove

Theorem 4. Let $0<p \leqq \infty$ and $f(t) \in L_{\rho}(-\infty, \infty)$ or $\in L_{p}(0, \infty)$, respectively, let $f(t)$ be approximated by functions $g_{\alpha}(z) \in G_{\alpha}$ or $\in G_{\alpha}^{(1 / 2)}$; let $\beta_{\alpha}$ be the actual $\left({ }^{18}\right)$ type of $g_{\alpha}(z)$, and let $c=\lim \inf \beta_{\alpha}(\alpha \rightarrow \infty)$ be finite. Then there is an element $F(z) \in G_{c}$ or $\in G_{c}^{(1 / 2)}$, respectively, such that $f(t) \equiv F(t)$ in $(-\infty, \infty)$ or in $(0, \infty)$.

By the hypothesis, there is a sequence $\left\{\alpha_{n}\right\}(n=1,2, \cdots)$ such that $g_{\alpha_{n}} \rightarrow f, n \rightarrow \infty$, and that the sequence $\left\{\beta_{n}\right\}$ of the actual types of the $g_{\alpha_{n}}(z)$ tends to $c$. Given $\epsilon>0$, we have $\beta_{n} \leqq c+\epsilon$ for $n \geqq A=A(\epsilon), g_{\alpha_{n}}(z) \in G_{c+\epsilon}$ or $G_{c+\epsilon}^{(1 / 2)}$, respectively, and

$$
\left|g_{\alpha_{m}}(t)-g_{\alpha_{n}}(t)\right|_{p} \rightarrow 0 \text { or }\left\|g_{\alpha_{m}}(t)-g_{\alpha_{n}}(t)\right\|_{p} \rightarrow 0
$$

as $m>n \rightarrow \infty$. By Lemma 3, the $g_{\alpha_{n}}(z)$ converge to a function $F(z)$ belonging to $G_{c+\epsilon}$ or $G_{c+\epsilon}^{(1 / 2)}$, respectively. We can take $\epsilon \rightarrow 0$. Evidently $F(t) \equiv f(t)$ which proves the theorem.

\section{The problem of best approximation.}

Theorem 5. Let $0<p \leqq \infty$ and $f(t) \in L_{p}(-\infty, \infty)$ or $\in L_{p}(0, \infty)$, respectively, and let $\alpha$ be fixed. There exists a function $g(z) \in G_{\alpha}$ or $\in G_{\alpha}^{(1 / 2)}$ of best approximation to $f(t)$, satisfying the inequality $|f(t)-g(t)|_{p} \leqq|f(t)-h(t)|_{p}$ or $\|f(t)-g(t)\|_{p} \leqq\|f(t)-h(t)\|_{p}$ for any $h(z) \in G_{\alpha}$ or $\in G_{\alpha}^{(1 / 2)}$, respectively. For $1<p<\infty, g_{\alpha}(z)$ is certainly unique.

Let $f(t) \in L_{p}(-\infty, \infty)$, and let $h_{0}(z)$ be an arbitrary element of $G_{\alpha, p}(\S 3)$.

(18) $\beta_{\alpha}=\lim \sup _{r \rightarrow \infty} \max _{|z|-r}\left(\log \left|g_{\alpha}(z)\right| / r\right)$. 
Denoting by $E$ the set of elements $h(z)$ of $G_{\alpha}$ such that $|f(t)-h(t)|_{p} \leqq A_{0}$ $=\left|f(t)-h_{0}(t)\right|_{p}$, by the argument of $\$ 3$ we deduce that, uniformly for $h(z) \in E$, $|h(z)|<A_{1} e^{\alpha|y|}$. By a well known argument( $\left.{ }^{19}\right)$ we can now deduce the required result, using the Lemmas $3,3^{\prime}$ and, in the proof of the last assertion of the theorem, Minkowski's inequality. The interval $(0, \infty)$ can be dealt with in a similar way.

THEOREM $5^{\prime}$. Let $f(t) \in L_{2}(-\infty, \infty)$. Then the sequence of best approximation is $\left\{g_{\alpha}(z)\right\}=\left\{D_{\alpha} f\right\}(0<\alpha \rightarrow \infty)$, where

$$
D_{\alpha} f=D_{\alpha}[f ; z]=\frac{1}{\pi} \int_{-\infty}^{\infty} \frac{\sin \alpha(t-z)}{t-z} f(t) d t
$$

is the Dirichlet singular integral.

By Lemma 4 , we have $D_{\alpha} f \in G_{\alpha}$. Let $F(u)$ be the Fourier transform of $f(t)$. It is well known $\left({ }^{20}\right)$ that

$$
D_{\alpha}[f ; x]=(2 \pi)^{-1 / 2} \int_{-\alpha}^{\alpha} F(u) e^{i x u} d u .
$$

Hence $\Phi(u)$, the Fourier transform of $f(x)-D_{\alpha} f$, vanishes for $|u|<\alpha$. By the Paley-Wiener $\left.{ }^{21}\right)$ theorem, $\Psi(u)$, the Fourier transform of $D_{\alpha} f-h(x)$, vanishes for $|u|>\alpha$ whenever $h(z) \in G_{\alpha, 2}$. Since the Fourier transform is isometric, we have

$$
\int_{-\infty}^{\infty}\left(f(x)-D_{\alpha} f\right)\left(\overline{D_{\alpha} f-h(x)}\right) d x=\int_{-\infty}^{\infty} \Phi(u) \bar{\Psi}(u) d u=0 .
$$

Consequently

$$
\int_{-\infty}^{\infty}|f(x)-h(x)|^{2} d x=\int_{-\infty}^{\infty}\left|f(x)-D_{\alpha} f\right|^{2} d x+\int_{-\infty}^{\infty}\left|D_{\alpha} f-h(x)\right|^{2} d x .
$$

The right side is greater than $\left|f(x)-D_{\alpha}\right|_{2}^{2}$ when $h(x)$ is different from $D_{\alpha} f$, which proves the theorem. It can be shown that there exists a constant $a \geqq 2$ such that, for $a<p<\infty$ and for $1<p<a(a-1)^{-1}, D_{\alpha} f$ is not a function of best approximation of $f(t)$.

10. A generalization of problem I. Let $C$ be a point set possessing the following properties:

(i) There exists an integral function $w=\Phi(z)$ which maps $C$ on the real axis of the $z$-plane in a one-to-one correspondence.

(ii) The inverse function $z=\Psi(w)$ is continuous in $(-\infty, \infty)$.

(19) J. L. Walsh, loc. cit., chapter 12.

(20) E.g., E. Hille, loc. cit. footnote 14, $\$ 5$.

(21) Fourier transforms in the complex domain, Amer. Math. Soc. Colloquium Publications, vol. 19, 1934, pp. 12-13. 
Again let $C^{\prime}$ be a point set satisfying (i) and, instead of (ii), the weaker condition

(ii) ${ }^{\prime} \Psi^{\prime}(w)$ exists and is finite for almost all $w$ in $(-\infty, \infty)$. From Theorems $3(a)$ and (b) we can deduce

THEOREM 6. Let $f(z)$ be bounded and uniformly continuous on $C$, or let, for $p=1,1 / 2,1 / 3, \cdots, f(z) \in L_{p}\left(C^{\prime}\right)$, that is to say, let $f(z)$ be measurable on $C^{\prime}$ and the integral

$$
\int_{C^{\prime}}|f(z)|^{p}|d z|=\int_{-\infty}^{\infty}|f(\Psi(w))| p\left|\Psi^{\prime}(w)\right||d w| \quad\left(p^{-1} \text { a positive integer }\right)
$$

be finite. Then there are integral functions $h_{\alpha}(z)$ such that, as $\alpha \rightarrow \infty$,

$$
\underset{z \text { on } C}{\text { ess. u.b. }}\left|f(z)-h_{\alpha}(z)\right| \rightarrow 0
$$

or

$$
\int_{C^{\prime}}\left|f(z)-h_{\alpha}(z)\right|^{p}|d z| \rightarrow 0,
$$

respectively, and $\left|h_{\alpha}(z)\right|<A_{\alpha} e^{\alpha|\Phi(z)|}$ or $\left\langle A_{\alpha}\left|\Phi^{\prime}(z)\right|^{1 / p} e^{\alpha|\Phi(z)|}\right.$.

Making use of Theorem 3(c), we can, in the general case $0<p<\infty$, construct integral functions $h_{\alpha}(z)$ satisfying (10.2), certainly if $C^{\prime}$ satisfies (i) and the following conditions:

(ii) $\Psi^{\prime}(w)$ is bounded in $(-\infty, \infty)$.

(iii) For some $r(0<r \leqq 1)$ and uniformly in $(-\infty, \infty)$,

$$
\left|\left(\Psi^{\prime}\left(w_{1}\right)\right)^{-1 / p}-\left(\Psi^{\prime}\left(w_{2}\right)\right)^{-1 / p}\right| \leqq A\left|w_{1}-w_{2}\right|^{r} .
$$

For instance, taking $w=\Phi(z)=z^{2}-2 i a, a>0$, and taking $C^{\prime}$ as the upper branch of the hyperbola $x y=a$, it will be seen that, for $\frac{1}{2} \leqq p<\infty, C^{\prime}$ has the required properties, with $r=1, A=(8 a)^{1 / 2}(4 a p)^{-1}$.

The University,

Edgbaston, Birmingham, England. 